\title{
A Time Scheduling Model of Logistics Service Supply Chain with Mass Customized Logistics Service
}

\author{
Weihua Liu, ${ }^{1}$ Yi Yang, ${ }^{1} X_{i a n g ~} \mathrm{Li}_{,}{ }^{2}$ Haitao $\mathrm{Xu}{ }^{1}$ and Dong Xie ${ }^{1}$ \\ ${ }^{1}$ School of Management, Tianjin University, Tianjin 300072, China \\ ${ }^{2}$ College of Economic and Social Development, Nankai University, Tianjin 300071, China \\ Correspondence should be addressed to Weihua Liu, lwhliu@yahoo.com.cn
}

Received 29 July 2012; Accepted 8 October 2012

Academic Editor: Xiaochen Sun

Copyright (c) 2012 Weihua Liu et al. This is an open access article distributed under the Creative Commons Attribution License, which permits unrestricted use, distribution, and reproduction in any medium, provided the original work is properly cited.

With the increasing demand for customized logistics services in the manufacturing industry, the key factor in realizing the competitiveness of a logistics service supply chain (LSSC) is whether it can meet specific requirements with the cost of mass service. In this case, in-depth research on the time-scheduling of LSSC is required. Setting the total cost, completion time, and the satisfaction of functional logistics service providers (FLSPs) as optimal targets, this paper establishes a time scheduling model of LSSC, which is constrained by the service order time requirement. Numerical analysis is conducted by using Matlab 7.0 software. The effects of the relationship cost coefficient and the time delay coefficient on the comprehensive performance of LSSC are discussed. The results demonstrate that with the time scheduling model in mass-customized logistics services (MCLSs) environment, the logistics service integrator (LSI) can complete the order earlier or later than scheduled. With the increase of the relationship cost coefficient and the time delay coefficient, the comprehensive performance of LSSC also increases and tends towards stability. In addition, the time delay coefficient has a better effect in increasing the LSSC's comprehensive performance than the relationship cost coefficient does.

\section{Introduction}

In the face of growing demand for customized logistics services, numerous logistics enterprises are not only providing customers with mass services, but are also beginning to meet the demand for customized services and are considering a change in their logistics service modes. Specifically, they try to provide mass customized logistics services (MCLS) instead of mass logistics services [1]. MCLS represents a significant development in logistics services, and a 
logistics company's capability to offer MCLS is crucial to enhance its market competitiveness. In the MCLS environment, to meet customized service demand and offer large-scale services, several logistics enterprises form a logistics service supply chain (LSSC) via union and integration [2,3]. LSSC is a new supply chain of which logistics service integrator (LSI) is the core enterprise. The basic structure of LSSC is functional logistics service provider $($ FLSP $) \rightarrow$ LSI $\rightarrow$ customer. FLSP is integrated by LSI when LSI builds the integrated logistics to customer. The main purpose of LSSC is to provide the flexible logistics service for manufacturing supply chain [3]. As the core enterprise of a LSSC, the LSI integrates the advantages of the FLSPs, such as various logistics processes and logistics service functions and then provides flexible logistics services to customers.

In such an environment, the key to improve the LSSC's competitiveness is its capability to offer customized logistics services with mass logistics costs. An in-depth research on the time scheduling of the LSSC is necessary to meet customers' requirements. However, the existing research on supply chain scheduling model has three deficiencies, which are discussed as follows.

First, the existing research on supply chain scheduling does not consider the feature that the operation time of suppliers may be delayed or compressed. It also ignores the fact that the customer's order is flexible in many cases, and that the order completion time may be delayed or compressed within an acceptable range. Thus, questions arise in expressing features of time delay or compression in model building and effects of time delay or compression on the comprehensive performance of LSSC.

Second, in most studies on supply chain scheduling, cost control is regarded as the primary scheduling goal. However, this is not always the case for an LSSC in the MCLS environment. The flexibility of the order completion time and the satisfaction of FLSPs are also significant. Expressing these objective functions in an optimization model and searching for a more reasonable method of solving this model are pertinent questions.

Third, the conclusions drawn from traditional manufacturing supply chain scheduling models may not be fully applicable to LSSC scheduling problems. Therefore, we will determine how LSI can use the time scheduling model proposed in this paper to better manage its LSSC.

This paper is organized as follows. Section 2 is the literature review in which the existing supply chain optimization scheduling models and methods are systematically summarized. Section 3 focuses on model building, in which a time scheduling optimization model of LSSC in the MCLS environment is established. Section 4 describes the model solution: a method of solving the multiobjective programming model. Section 5 presents numerical analysis, including a discussion of the influence of relevant parameters on LSSC's scheduling performance. Section 6 provides management insights and recommendations to improve actual operation of LSSC. The last section presents the conclusions and future research directions in this field.

\section{Literature Review}

Most studies on supply chain scheduling have focused on the manufacturing industry and have achieved significant results. Many earlier studies discussed job shop scheduling within a single enterprise (see, e.g., $[4,5]$ ). They are primarily interested in the arrangement of processing procedures and the order operation sequence. Kreipl and Pinedo [6] introduced what is planning and what is scheduling and gave an overview of the various planning and scheduling models in general. Several scholars wrote about the coordination of the assembly 
system in manufacturing enterprise (see, e.g., [7]). But in early studies, many researches emphases were focused within one manufacturing enterprise and did not pay much attention to the roles outside the enterprise. As is known to all, customer is the most important role in the competitive market. Philipoom [8] transferred his attention to the most important part, which is the customers' requirements and their feelings. The dilemma for the manufacturing manager when planning the lead-times was discussed. He took delivery reliability and time as the vital factors in scheduling. The idea of thinking highly of customer satisfactory is worthy of our research. A systematic study of the supply chain scheduling model had been established by Hall and Potts [9] and from then on planning and scheduling problems began getting more attention.

However, studies on the supply chain scheduling with the mass-customization production mode are a relatively new development (see, e.g., $[10,11]$ ). The contents of these studies included the application of postponement strategy, the positioning of customer order decoupling point (CODP), and the time scheduling problem. In terms of the supply chain time scheduling, several scholars examined the dominant contradictions analysis and its optimization solution of supply chain scheduling in mass customization (see, e.g., $[12,13]$ ). Yao and $\mathrm{Pu}$ [13] introduced a dynamic and multiobjective optimization model to balance the contradiction between scale production effect and customized demand. They considered the delay coefficient but did not give out the time delay punishment in mathematical expression. Other scholars studied the differences between the ideal timetables of the different supply chain members and that of customer demand, and then explored ways to solve this discrepancy (see, e.g., [14]). From the perspective of integrated supply chain production planning and scheduling process, Mishra et al. [15] designed a mixed integer programming model. Moreover, in the scheduling of manufacturing supply chain, the main objective of scheduling optimization is supply chain cost (see, e.g., [16-18]). Most studies assumed that the order completion time or the delivery time of suppliers was fixed. However, in a number of cases, as an index reflecting supply chain agility, the order time requirements may be changed (see, e.g., [19-21]). Thus, considering the influence of time compression or delay on the scheduling results is necessary.

Similar to studies on manufacturing supply chain, research on the service supply chain mainly focuses on service process scheduling (see, e.g., [22, 23]), order assignment scheduling (see, e.g., $[3,24]$ ), and so forth. Time scheduling, although is a significant part of LSSC scheduling, has not received sufficient attention. Time scheduling is a key aspect of the service supply chain operation. It is directly related to whether the logistics service can be completed successfully based on customer requirements. Therefore, examining time scheduling of LSSC is necessary.

This paper aims to address these issues and to evaluate the time scheduling model of LSSC in the MCLS environment. The main contributions of this study are listed as follows.

(1) The effects of time delay and compression of the customer order on LSSC's comprehensive performance are analyzed. Time delay in the order requirements contributes to an increase in the satisfaction of FLSPs. However, their satisfaction level remains stable after it reaches a certain value. Moreover, the extent of time compression is also limited.

(2) The effect of the relationship cost coefficient on the comprehensive performance of LSSC is discussed. With the increase of LSI's relationship cost coefficient, the comprehensive performance of LSSC also increases. This comprehensive performance cannot be infinitely enhanced, but remains stable upon reaching a certain value. 
(3) LSI can improve the comprehensive performance of LSSC by both the relationship cost coefficient and time delay. However, compared with the relationship cost coefficient, using time delay is a superior way to improve the comprehensive performance.

\section{Time Scheduling Model of LSSC}

\subsection{Model Assumptions and Variables}

A two-echelon LSSC is assumed to consist of one LSI and several FLSPs. The logistics service comprises multiple service processes. Every service process is completed by many cooperative FLSPs. Each FLSP's service capability is different from that of the others; different FLSPs have varying normal completion times for the same work. Table 1 presents the notations of the model.

The other assumptions of the model are as follows.

(1) The $(i+1)$ th service process cannot begin until the $i$ th service process is finished.

(2) In each service process, the quantity of service orders assigned by LSI to FLSP is assumed given. FLSP can only complete these orders based on the order quantity assigned by LSI. The service cost of FLSP is equal to the service cost per unit time multiplied by service time.

(3) The normal service time of FLSP refers to the typical length of time needed to complete a task using its capability without considering any time delay or compression. While working within the normal time, the satisfaction of FLSP is at its highest, and the FLSP does not have to pay any additional cost.

(4) In each service process, time delay or compression to complete an order produces additional costs. The costs of time delay or compression per unit time are the same.

(5) All the service processes are outsourced to FLSPs by LSI and are completed by the former. The model does not consider the operation cost of LSI.

(6) The assignment of the service orders is finished. And the CODP has been decided. The model does not consider the location problem of the CODP.

(7) $T_{i j}$ means the normal service time of the $j$ th FLSP in the $i$ th service process, which is a parameter decided by the technology of the corresponding FLSP and differs from each other. In the section of numerical analysis (see Section 5), it is a definitive parameter. In practice, the normal service time of finishing a specific order is usually definitive, but not stochastic.

\subsection{Model Building}

Considering multiple goals in LSSC time scheduling is necessary. To minimize the total cost incurred in LSSC, to minimize the difference between the expected and the actual time of completing the service order, and to maximize the satisfaction of FLSPs, this paper establishes a time-scheduling model that is constrained by the service order time requirement. The modeling process is as follows. 
Table 1: Notations for the model.

\begin{tabular}{|c|c|}
\hline Notations & Description \\
\hline$C_{i j}$ & $\begin{array}{l}\text { The normal service cost per unit time of the } j \text { th FLSP in the } i \text { th service process, which is } \\
i=1,2,3, \ldots, I_{0}, j=1,2,3, \ldots, J_{0} \text {, the same as below }\end{array}$ \\
\hline$C_{i j}^{\text {ext }}$ & The additional service cost per unit time of the $j$ th FLSP in the $i$ th service process \\
\hline$C_{i}^{\text {ext }}$ & $\begin{array}{l}\text { When the } i \text { th service process is delayed, the delay cost per unit time incurred in the } \\
(i+1) \text { th service process }\end{array}$ \\
\hline$p_{i}^{\text {ext }}$ & $\begin{array}{l}\text { When the time of the } i \text { th service process is compressed, the time compression cost per } \\
\text { unit time incurred in the }(i+1) \text { th service process }\end{array}$ \\
\hline$T_{i j}$ & The normal service time of the $j$ th FLSP in the $i$ th service process \\
\hline$T_{i j}^{\text {ext }}$ & The additional service time of the $j$ th FLSP in the $i$ th service process \\
\hline$T_{i}^{\exp }$ & The expected operation time for the $i$ th service process set by LSI \\
\hline$U_{i j}$ & The satisfaction of the $j$ th FLSP in the $i$ th service process \\
\hline$\phi_{i j}$ & Weights of the $j$ th FLSP's satisfaction in the $i$ th service process \\
\hline$\beta_{i}$ & Time delay coefficient for the $i$ th service process set by LSI \\
\hline$T_{i}^{*}$ & $\begin{array}{l}\text { The upper limit of time delay or compression incurred in the }(i-1) \text { th service process, } \\
\text { which can be endured by the } i \text { th service process }\end{array}$ \\
\hline$U_{i j}^{0}$ & The lower limit of the satisfaction of the $j$ th FLSP in the $i$ th service process \\
\hline$R$ & $\begin{array}{l}\text { The adjustment coefficient of the time delay or compression, in which } R>0 \text { means that } \\
\text { the operation time is delayed. } R<0 \text { indicates that the operation time is compressed }\end{array}$ \\
\hline$Z_{1}$ & The total cost of LSSC \\
\hline$Z_{2}$ & The total delivery time of all processes in LSSC \\
\hline$Z_{3}$ & The total satisfaction of FLSPs in LSSC \\
\hline$Z_{1}^{\min }$ & $\begin{array}{l}\text { The minimum of the objective function } Z_{1} \text { when the objective functions } Z_{2} \text { and } Z_{3} \text { are } \\
\text { not considered }\end{array}$ \\
\hline$Z_{2}^{\min }$ & $\begin{array}{l}\text { The minimum of the objective function } Z_{2} \text { when the objective functions } Z_{1} \text { and } Z_{3} \text { are } \\
\text { not considered }\end{array}$ \\
\hline$Z_{3}^{\min }$ & $\begin{array}{l}\text { The minimum of the objective function } Z_{3} \text { when the objective functions } Z_{1} \text { and } Z_{2} \text { are } \\
\text { not considered }\end{array}$ \\
\hline$Z_{2}^{\max }$ & $\begin{array}{l}\text { The maximum of the objective function } Z_{2} \text { when the objective functions } Z_{1} \text { and } Z_{3} \text { are } \\
\text { not considered }\end{array}$ \\
\hline$Z_{3}^{\max }$ & $\begin{array}{l}\text { The maximum of the objective function } Z_{3} \text { when the objective functions } Z_{1} \text { and } Z_{2} \text { are } \\
\text { not considered }\end{array}$ \\
\hline C & The relationship cost coefficient of the LSI, $C>1$ \\
\hline Z & $\begin{array}{l}\text { The objective function synthesized by } Z_{2} \text { and } Z_{3} \text {, which is also called the } \\
\text { comprehensive performance of LSSC }\end{array}$ \\
\hline$K_{1}$ & $\begin{array}{l}\text { Weight coefficients of the objective function } Z_{2} \text { in the comprehensive performance of } \\
\text { LSSC }\end{array}$ \\
\hline$K_{2}$ & $\begin{array}{l}\text { Weight coefficients of the objective function } Z_{1} \text { in the comprehensive performance of } \\
\text { LSSC }\end{array}$ \\
\hline$F_{i j}$ & The $j$ th FLSP in the $i$ th service process \\
\hline
\end{tabular}


We set that $[f(x)]^{+}=\max \{0, f(x)\}$.

Then

$$
\begin{aligned}
\min Z_{1}= & \sum_{i=1}^{I_{0}} \sum_{j=1}^{J_{0}}\left(C_{i j} T_{i j}+C_{i j}^{\text {ext }}\left|T_{i j}^{\text {ext }}\right|\right)+\sum_{i=1}^{I_{0}}\left(\max _{j=1}^{J_{0}}\left[T_{i j}+T_{i j}^{\text {ext }}-T_{i}^{\text {exp }}\right]^{+}\right) C_{i}^{\text {ext }} \\
& +\sum_{i=1}^{I_{0}}\left(\min _{j=1}^{J_{0}}\left[T_{i}^{\text {exp }}-T_{i j}-T_{i j}^{\text {ext }}\right]^{+}\right) p_{i}^{\text {ext }}, \\
\min Z_{2}= & \sum_{i=1}^{I_{0}}\left(\min _{j=1}^{J_{0}}\left[T_{i}^{\text {exp }}-T_{i j}-T_{i j}^{\text {ext }}\right]^{+}\right), \\
\max Z_{3}= & \sum_{i=1}^{I_{0}} \sum_{j=1}^{J_{0}}\left(1-\frac{\left|T_{i j}-T_{i}^{\text {exp }}\right|}{T_{i j}}\right) \frac{T_{i j} C_{i j}}{\left(T_{i j} C_{i j}+\left|T_{i j}^{\text {ext }}\right| C_{i j}^{\text {ext }}\right)} \phi_{i j},
\end{aligned}
$$

subject to $\quad T_{i j}+T_{i j}^{\mathrm{ext}} \leq\left(1+\beta_{i} R\right) T_{i}^{\mathrm{exp}}$,

$$
\begin{aligned}
& \left|T_{i}^{\text {exp }}-T_{i j}-T_{i j}^{\text {ext }}\right| \leq T_{i+1}^{*}, \\
& \left(1-\frac{\left|T_{i j}-T_{i}^{\text {exp }}\right|}{T_{i j}}\right) \frac{T_{i j} C_{i j}}{\left(T_{i j} C_{i j}+\left|T_{i j}^{\text {ext }}\right| C_{i j}^{\text {ext }}\right)} \geq U_{i j}^{0} .
\end{aligned}
$$

In (3.1), the objective function $Z_{1}$ is made to minimize LSSC total operation cost. The first part of (3.1) represents the total operation cost of all processes in the LSSC. The second part of (3.1) represents the time delay cost incurred in the next process, which is caused by the previous process. The third part of (3.1) represents the cost of time compression incurred in the next process, which is caused by the previous process too.

In (3.2), the objective function $Z_{2}$ makes the service order completed on time as much as possible.

In (3.3), the objective function $Z_{3}$ is made to maximize the weighted satisfaction of all FLSPs. This indicator consists of two parts: one is the satisfaction with operation time and the other is related to the cost. In $Z_{3}$, the first part $\left(1-\left|T_{i j}-T_{i}^{\exp }\right| / T_{i j}\right)$ represents the proximity degree between the normal operation time and the order expected time of the $j$ th FLSPs in the $i$ th service process, which represents the satisfaction in the time aspect. The second part $T_{i j} C_{i j} /\left(T_{i j} C_{i j}+\left|T_{i j}^{\text {ext }}\right| C_{i j}^{\text {ext }}\right)$ means the proportion of the normal operation cost in the total cost of the $j$ th FLSP in the $i$ th service process, which represents the satisfaction in the cost aspect.

In (3.4), the first constraint represents the requirements of FLSP's order completion time proposed by the LSI. Specifically, it requires that the actual completion time must be less than a certain multiple of the expected time. This multiple is determined by the product of $\beta_{i}$ and $R$, which represents the limits that LSI can endure the time delay or compression. The second constraint means that the difference between the actual order completion time and the expected time cannot exceed the upper limit of the time variation (time compression or delay) 
incurred in the $(i-1)$ th service process, which can be endured by the $i$ th service process. This is a strong constraint that must be observed because during the entire service provision process, the continued link of operation time exists between the upstream and downstream service processes. The starting time of each process should meet certain time requirements. The third constraint means that the satisfaction of each FLSP must be more than the lower limit that they can accept.

\section{Model Solution}

\subsection{Simplifying the Multiobjective Programming Model}

The model described has three objectives and three constraints. It is a typical multiobjective programming problem. Multiobjective programming problems have numerous mature solutions, such as the evaluation function method (including linear weighting method, reference target method, maximin method), goal programming method, delaminating sequence method, interactive programming method, and subordinate function method. For the specific issues, it is necessary to choose an appropriate solution method to solve the practical problems. This paper is based on the MCLS environment, so we should consider not only the cost target but also the customized target. In time scheduling, LSI tends to conform to the customized time requirements and set corresponding requirements related to order completion time for their FLSPs. Each FLSP's satisfaction will directly determine the quality and the possibility of order completion. Thus, order completion time and FLSPs' satisfaction are two critical goals. Operation cost is often not the primary consideration. Completing logistics service orders with the minimum cost is not required; the total cost of LSSC must be maintained within a certain range. Besides, to build and maintain a good relationship with FLSPs, LSI is usually willing to make a certain amount of cost concessions. Considering these actual situations, we introduce the following parameter called the relationship cost coefficient $C(C>1)$ into our model and use it to represent the cost augmentation limits (4.1).

$$
Z_{1}<Z_{1}^{\min } \times C
$$

Thus, (4.1) can be regarded as a new constraint and be combined with (3.4) to form the new constraints. The original model then becomes a twin-goal programming problem. Given that a certain degree of conflict and incommensurability occurs among the targets in multiobjective decision-making problems, determining an absolute optimal solution is difficult. In this paper, we choose the commonly used linear weighting method to solve our model, and attempt to transform this multiobjective programming model into a singleobjective programming model.

The objective function $Z_{2}$ and the objective function $Z_{3}$ mean to make the service order completed on time as much as possible and to maximize the satisfaction of FLSPs, respectively. Therefore, each of them has to be normalized. $Z_{2}$ and $Z_{3}$ are thus divided by the possible maximum value $Z_{2}^{\max }$ and $Z_{3}^{\max }$, respectively, and their corresponding results are added up. The synthesized objective function is as follows:

$$
\max Z=K_{2} \times \frac{Z_{3}}{Z_{3}^{\max }}-K_{1} \times \frac{Z_{2}}{Z_{2}^{\max }}
$$


In (4.2), $K_{1}$ and $K_{2}$ represent the weights of $Z_{2}$ and $Z_{3}$, respectively, which are determined by the linear weighting method. Consider the following:

$$
\begin{array}{ll}
\max & Z=K_{2} \times \frac{Z_{3}}{Z_{3}^{\max }}-K_{1} \times \frac{Z_{2}}{Z_{2}^{\max },} \\
\text { subject to } \quad & T_{i j}+T_{i j}^{\text {ext }} \leq\left(1+\beta_{i} R\right) T_{i}^{\text {exp }}, \\
& \left|T_{i}^{\text {exp }}-T_{i j}-T_{i j}^{\text {ext }}\right| \leq T_{i+1}^{*}, \\
& \left(1-\frac{\left|T_{i j}-T_{i}^{\text {exp }}\right|}{T_{i j}}\right) \frac{T_{i j} C_{i j}}{\left(T_{i j} C_{i j}+\left|T_{i j}^{\text {ext }}\right| C_{i j}^{\text {ext }}\right)} \geq U_{i j}^{0}, \\
& Z_{1}<Z_{1}^{\min } C .
\end{array}
$$

Here,

$$
\begin{aligned}
Z_{1}= & \sum_{i=1}^{I_{0}} \sum_{j=1}^{J_{0}}\left(C_{i j} T_{i j}+C_{i j}^{\text {ext }}\left|T_{i j}^{\text {ext }}\right|\right)+\sum_{i=1}^{I_{0}}\left(\max _{j=1}^{J_{0}}\left[T_{i j}+T_{i j}^{\text {ext }}-T_{i}^{\text {exp }}\right]^{+}\right) C_{i}^{\text {ext }} \\
& +\sum_{i=1}^{I_{0}}\left(\min _{j=1}^{J_{0}}\left[T_{i}^{\text {exp }}-T_{i j}-T_{i j}^{\text {ext }}\right]^{+}\right) p_{i}^{\text {ext }}, \\
Z_{2}= & \sum_{i=1}^{I_{0}}\left(\min _{j=1}^{J_{0}}\left[T_{i}^{\text {exp }}-T_{i j}-T_{i j}^{\text {ext }}\right]^{+}\right), \\
Z_{3}= & \sum_{i=1}^{I_{0}} \sum_{j=1}^{J_{0}}\left(1-\frac{\left|T_{i j}-T_{i}^{\text {exp }}\right|}{T_{i j}}\right) \frac{T_{i j} C_{i j}}{\left(T_{i j} C_{i j}+\left|T_{i j}^{\text {ext }}\right| C_{i j}^{\text {ext }}\right)} \phi_{i j} .
\end{aligned}
$$

\subsection{Using the Genetic Algorithm to Solve the Multiobjective Programming Problem}

The genetic algorithm (GA) is an effective method of searching for the optimal solution by simulation of the natural selection process. It uses multiple starting points to begin the search; it has global search capability. Similar to the natural evolutionary process, the computational process of genetic algorithms is iterative, which means that it includes selection, crossover, and mutation. For reference, GA is described in [25].

\section{Numerical Analysis}

This section first illustrates the validity of the model via a numerical analysis, and then explores the influence of relevant parameters on time scheduling results. Additionally, some effective recommendations are given for use in the actual operation-based on numerical analysis. 


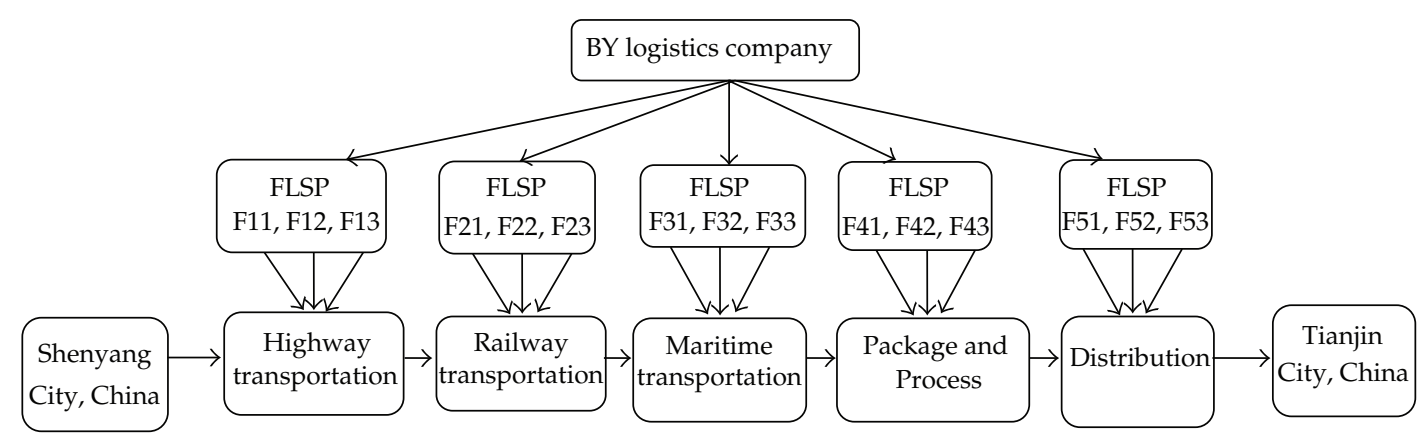

Figure 1: The logistics service process of one order of BY Company.

\subsection{Numerical Data Description and Basic Results}

BY is a logistics company based in northern China. It is a logistics integrator that has integrated several third-party logistics firms. This company has received an order requiring it to deliver goods from Shenyang City to Tianjin City. During delivery, five service processes are needed: road transportation, rail transportation, maritime transportation, packaging and processing, and distribution. The CODP has been decided and it will be at the 4 th service process which is packaging process. Obviously, the processes before the CODP are provided with mass operations and the processes after the CODP are provided with customized operations. Each service process is to be completed by three FLSPs (see Figure 1).

The service capability of each FLSP is different from that of the others because the normal operation times $T_{i j}$ vary when different FLSPs complete the same order. BY company, the LSI, has set an expected operation time $T_{i}^{\exp }$ for each order in every process. The parameters of each process and of each FLSP are different (Tables 2 and 3). We assume that $i$ be the service process, $i=1,2,3,4,5, j$ represents the number of FLSPs in each process, so that $j=1,2,3 . C_{i j}$ represents the normal service cost per unit time of the $j$ th FLSP in the $i$ th process.

In the model solving, genetic algorithms and Matlab 7.0 software are used. Assuming the genetic population to be 200 , the hereditary algebra to be 400 , the adjustment coefficient $R=1$, and the relationship cost coefficient $C=1.2$, then the calculation result of comprehensive performance $Z$ is equal to 0.6802 . The time parameters of each FLSP in every process are shown in Table 4.

We can find that among the 15 FLSPs, seven completed the order with time compression $\left(T_{i j}^{\exp }<0\right)$, and eight completed the order with time delay $\left(T_{i j}^{\exp }>0\right)$.

\subsection{The Effect of Time Delay Coefficient on LSSC Scheduling Results}

In practice, responding rapidly to customized demand is one of the main features of the masscustomized LSSC. It is often the case that the LSI requires its FLSPs to compress their service time frequently to respond to the customer's demand. Therefore, the order is completed ahead of the expected time, which is reflected in the model as $\beta_{i} R<0$. In some cases, if the time requirement of the order is not that urgent, LSI may permit its FLSPs to delay their work. That is, the completion time is later than the expected time, which is reflected in the model as $\beta_{i} R>0$. The permitted order completion time is directly related to the difficulty faced by FLSPs in completing the order. It has a direct impact on the cost of these FLSPs. To describe 
Table 2: Basic data (3.1).

\begin{tabular}{lccc}
\hline$C_{i j}$ & $j=1$ & $j=2$ & $j=3$ \\
\hline$i=1$ & 5 & 6 & 5.5 \\
$i=2$ & 7 & 8 & 8.5 \\
$i=3$ & 11 & 12 & 10.5 \\
$i=4$ & 15 & 18 & 14.5 \\
$i=5$ & 6 & 5 & 7.5 \\
\hline$C_{i j}^{\text {ext }}$ & $j=1$ & $j=2$ & $j=3$ \\
\hline$i=1$ & 5.5 & 6.5 & 6.5 \\
$i=2$ & 8 & 9 & 12 \\
$i=3$ & 12 & 14 & 16 \\
$i=4$ & 16 & 20 & 8 \\
$i=5$ & 7 & 6 & $j=3$ \\
\hline$T_{i j}$ & $j=1$ & $j=2$ & 6 \\
\hline$i=1$ & 8 & 7 & 5 \\
$i=2$ & 7 & 6 & 9.5 \\
$i=3$ & 10 & 9 & 11 \\
$i=4$ & 12 & 10 & 10 \\
$i=5$ & 8 & 9 & $j=3$ \\
\hline$U_{i j}^{0}$ & $j=1$ & $j=2$ & 0.3 \\
\hline$i=1$ & 0.4 & 0.5 & 0.55 \\
$i=2$ & 0.5 & 0.6 & 0.55 \\
$i=3$ & 0.4 & 0.5 & 0.3 \\
$i=4$ & 0.3 & 0.3 & 0.3 \\
$i=5$ & 0.3 & 0.25 & $j=3$ \\
\hline$\phi_{i j}$ & $j=1$ & $j=2$ & 0.4 \\
\hline$i=1$ & 0.4 & 0.3 & 0.3 \\
$i=2$ & 0.3 & 0.4 & 0.2 \\
$i=3$ & 0.5 & 0.3 & 0.5 \\
$i=4$ & 0.3 & 0.2 & 0.3 \\
$i=5$ & 0.4 & 0.3 &
\end{tabular}

Table 3: Basic data (3.2).

\begin{tabular}{|c|c|c|c|c|c|}
\hline$C_{i}^{\text {ext }}$ & $\begin{array}{c}C_{1}^{\text {ext }} \\
2\end{array}$ & $\begin{array}{c}C_{2}^{\text {ext }} \\
4\end{array}$ & $\begin{array}{c}C_{3}^{\text {ext }} \\
6\end{array}$ & $\begin{array}{c}C_{4}^{\text {ext }} \\
8\end{array}$ & $\begin{array}{c}C_{5}^{\text {ext }} \\
3\end{array}$ \\
\hline$p_{i}^{\mathrm{ext}}$ & $\begin{array}{c}p_{1}^{\mathrm{ext}} \\
3\end{array}$ & $\begin{array}{c}p_{2}^{\mathrm{ext}} \\
5\end{array}$ & $\begin{array}{c}p_{3}^{\text {ext }} \\
7\end{array}$ & $\begin{array}{c}p_{4}^{\text {ext }} \\
9\end{array}$ & $\begin{array}{c}p_{5}^{\text {ext }} \\
4\end{array}$ \\
\hline$T_{i}^{\exp }$ & $\begin{array}{c}T_{1}^{\exp } \\
7 \\
\end{array}$ & $\begin{array}{c}T_{2}^{\exp } \\
6 \\
\end{array}$ & $\begin{array}{c}T_{3}^{\exp } \\
8 \\
\end{array}$ & $\begin{array}{c}T_{2}^{\exp } \\
14 \\
\end{array}$ & $\begin{array}{c}T_{5}^{\exp } \\
8\end{array}$ \\
\hline$\beta_{i}$ & $\begin{array}{c}\beta_{1} \\
0.1\end{array}$ & $\begin{array}{c}\beta_{2} \\
0.2\end{array}$ & $\begin{array}{c}\beta_{3} \\
0.2\end{array}$ & $\begin{array}{c}\beta_{4} \\
0.15\end{array}$ & $\begin{array}{c}\beta_{5} \\
0.15\end{array}$ \\
\hline$T_{i}^{*}$ & $\begin{array}{c}T_{2}^{*} \\
1\end{array}$ & $\begin{array}{c}T_{3}^{*} \\
2\end{array}$ & $\begin{array}{c}T_{4}^{*} \\
3\end{array}$ & $\begin{array}{c}T_{5}^{*} \\
3\end{array}$ & $\begin{array}{c}T_{6}^{*} \\
2\end{array}$ \\
\hline
\end{tabular}

the two situations, we discuss the influence of the time delay (or compression) coefficient on the satisfaction of LSSC and on the order completion time. We use the synthesized objective function $Z$ to denote the comprehensive performance of LSSC. Keeping the model parameters unchanged and changing only the value of adjustment coefficient $R$, we obtain the calculation results of $Z$. The results are presented in Table 5 .

When the calculation results in Table 5 are plotted, a curve is shown in Figure 2. 
Table 4: Calculation results.

\begin{tabular}{llcc}
\hline Process $i$ & $F_{i j}$ & $T_{i j}^{\text {exp }}$ & The total operation time of each FLSP \\
\hline \multirow{3}{*}{$i=1$} & $F_{11}$ & -0.4181 & 7.5819 \\
& $F_{12}$ & 0.0223 & 7.0223 \\
& $F_{13}$ & 0.0204 & 6.0204 \\
\hline \multirow{3}{*}{$\begin{array}{l}* \\
*\end{array}$} & $F_{21}$ & 0.0287 & 7.0287 \\
& $F_{22}$ & -0.0039 & 5.9961 \\
& $F_{23}$ & 0.0001 & 5.0001 \\
\hline \multirow{3}{*}{3} & $F_{31}$ & -0.4028 & 9.5972 \\
& $F_{32}$ & -0.0014 & 8.9986 \\
& $F_{33}$ & -0.0297 & 9.4703 \\
\hline \multirow{3}{*}{4} & $F_{41}$ & 0.0087 & 12.0087 \\
& $F_{42}$ & 4.0001 & 14.0001 \\
& $F_{43}$ & 0.0363 & 11.0363 \\
\hline \multirow{3}{*}{5} & $F_{51}$ & -0.0151 & 7.9849 \\
& $F_{52}$ & 0.0110 & 9.0110 \\
& $F_{53}$ & -0.8012 & 9.1988
\end{tabular}

Table 5: The effect of time delay (compression) coefficient on the comprehensive performance of LSSC.

\begin{tabular}{lc}
\hline$R$ & $Z$ \\
\hline 2.4 & 0.7019 \\
2 & 0.7019 \\
1.6 & 0.6948 \\
1.2 & 0.6827 \\
1 & 0.6802 \\
0.8 & 0.6671 \\
0.4 & 0.6615 \\
0 & 0.6272 \\
-0.2 & 0.5999 \\
-0.4 & 0.5738 \\
-0.6 & 0.4429 \\
-0.8 & 0.3941 \\
-0.85 & 0.1175 \\
\hline
\end{tabular}

\subsubsection{Analysis of the Overall Change Trend}

From Figure 2, we can observe that along with the increase of $R$ (from negative to positive values), the synthesized objective function $Z$ rises gradually, which indicates that the satisfaction of LSSC increases and remains stable after reaching a certain value. The slope in the delay part is smaller; using the time delay strategy, the margin of increase in the LSSC's comprehensive performance is slow. However, the slope of the curve in the time compression part is bigger than that in the time delay part. Hence, the influence on the comprehensive performance of LSSC is greater. It is due to that along with the increase of $R$, these FLSPs can get time relaxation, but they are required to pay the delay cost at the same time. In addition, their additional time is occupied by the order, thus they could not use it to finish other service orders. And the more time relaxation is offered, the effect described above is larger. Therefore, $Z$ value is shown marginal decrease with the increasing of adjustment 


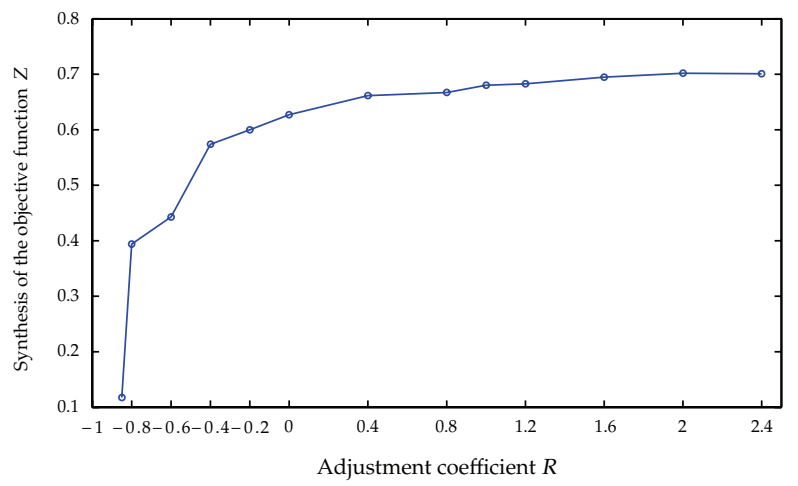

Figure 2: Curve of $Z$ changed with $R$.

coefficient $R$. Based on these facts, it can be inferred that in practice, before the LSI requires its FLSPs to operate with time compression (to shorten their operation time in finishing the same order), it is necessary to carefully weigh the advantages and disadvantages. Furthermore, following certain measures (such as offering cost compensation) to minimize the decline in the FLSPs' satisfaction is necessary.

To further analyze the influence on the LSSC performance caused by the time delay (or time compression) coefficient, Section 5.2.2 and Section 5.2.3 cover the relevant conclusions drawn from the different parts of Figure 2.

\subsubsection{Analysis of the Time Compression Part}

Figure 3 shows the curve of $Z$ changed with $R$ when operation time is compressed. When $R<0$, the LSSC is in the operation status of time compression. Figure 3 indicates that with the increase of the absolute value of $R, Z$ decreases, which means that the performance of LSSC is declining. In the part of $R$ decreasing from 0 to -0.9 , from the slope of the curve (Figure 3), the $Z$ value decreases by a much larger margin (from 0.6272 to 0.1175 ). Thus, if FLSPs are required to operate in the time compression state, their total satisfaction will decline sharply. Moreover, a greater increase in time compression indicates a greater decline in the satisfaction of FLSPs. In our numerical example, if $R<-0.9$, no solution in the model occurs, which means LSSC has collapsed and can no longer operate. This also shows that in practice, the order completion time of FLSP cannot be compressed infinitely. Upon reaching a certain point, the FLSP's operation time can no longer be compressed. When working within this limitation time, the comprehensive performance of LSSC is at its lowest.

\subsubsection{Analysis of the Time Delay Part}

Figure 4 shows the curve of $Z$ changed with $R$ when operation time is delayed. When $R>0$, LSSC is in the operation status of time delay. Figure 4 indicates that along with the increase of $R, Z$ also increases. That is, the comprehensive performance of LSSC continues to increase. However, when $R>0, Z$ only increases slightly. The range of increase of $Z$ is far less than the range of its decline when operation time is delayed or compressed at the same extent. This occurs because in the case of time delay, FLSPs must pay the penalty cost. In addition, the same with that in Figure 2, the $Z$ value is shown that marginal decrease with the increasing of adjustment coefficient $R$, the more time relaxation is offered, the more the service capacities 


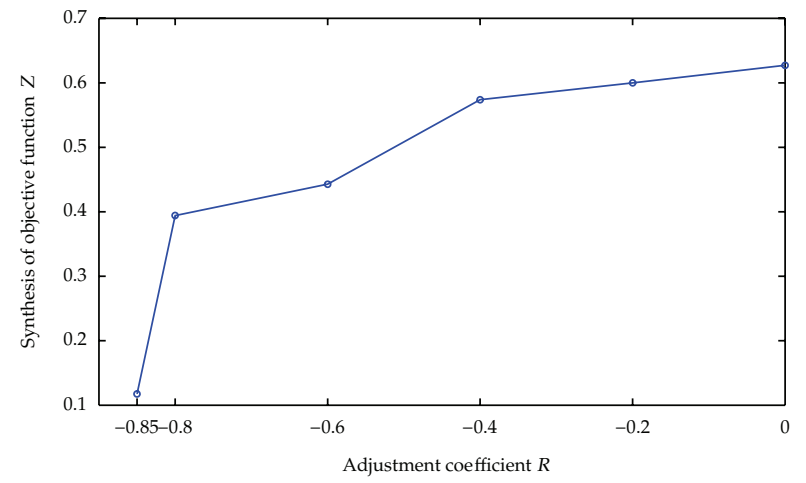

Figure 3: Curve of $Z$ changed with $R$ when operation time is compressed.

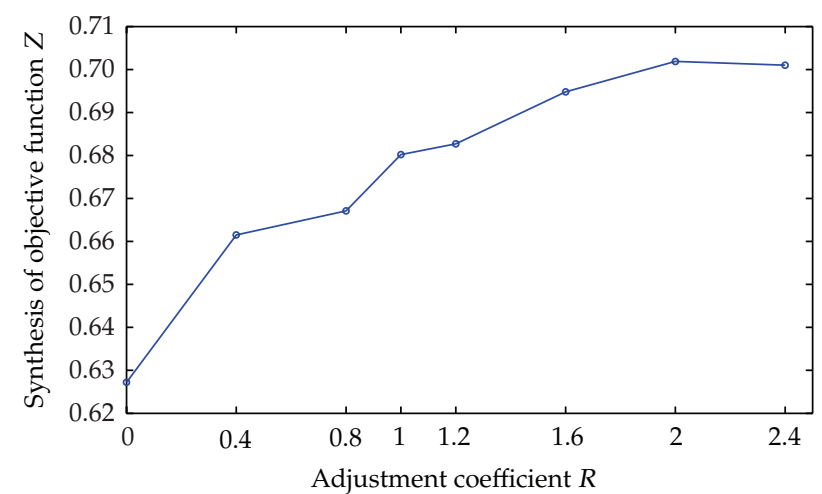

Figure 4: Curve of $Z$ changed with $R$ when operation time is delayed.

of FLSPs are occupied and cannot be used to complete other service orders. For the reasons mentioned, FLSPs are generally unwilling to delay their completion time.

Figure 4 illustrates that $Z$ remains unchanged after $R>2$, which shows that the time delay has reached the upper limit. Increasing the time delay coefficient is of no use to improve the $Z$ value anymore. In other words, $Z$ has reached the maximum at $R=2$. It also explains that in practice, the service time cannot be delayed infinitely.

In summary, allowing the FLSPs to delay the order completion time to a certain extent will contribute to LSCC performance. However, constrained by the time connections between the upstream and downstream service processes, the LSSC's performance improvement faces an upper limit.

\subsection{The Effect of Relationship Cost Coefficient of LSI on LSSC Scheduling Results}

The relationship cost coefficient is introduced into the model solving approach, and the cost of LSI is allowed to increase appropriately. In this part, we explore the effect of the LSI's relationship cost coefficient on LSSC scheduling results to provide a theoretical basis for the LSI's decision-making. 


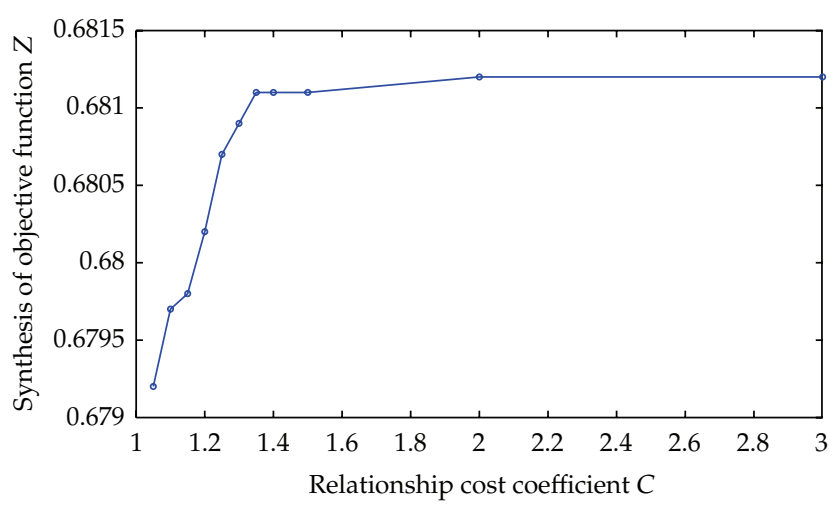

Figure 5: Curve of $Z$ changed with $C$.

Table 6: The effect of $C$ on $Z$.

\begin{tabular}{cccccccccccc}
\hline$C$ & 0.05 & 0.1 & 0.15 & 0.2 & 0.25 & 0.3 & 0.35 & 0.4 & 0.5 & 1 & 2 \\
\hline$Z$ & 0.6792 & 0.6797 & 0.6798 & 0.6802 & 0.6807 & 0.6809 & 0.6811 & 0.6811 & 0.6811 & 0.6812 & 0.6812 \\
\hline
\end{tabular}

The relationship cost coefficient is made by LSI. Its size will have a direct influence on the FLSP's satisfaction and on the overall performance of LSSC. To explore the relationship between the synthesized objective function and the cost relationship coefficient, we assign different values to $C$, and then obtain the corresponding $Z$ (Table 6 and Figure 5).

Figure 5 shows the curve of $Z$ changed with $C$. It indicates that along with the increase of $C, Z$ also increases and ultimately tends to stabilize at 0.6812 . Along with the increase of the LSI's relationship cost coefficient, the comprehensive performance of LSSC increases as well. However, this improvement is not unlimited. Instead, it remains stable after reaching a certain value due to the mandatory requirement on service time that FLSPs must meet. As mentioned, the service time cannot be delayed or compressed without limit. Thus, when the cost augmentation increases to a certain level, continuing to increase the cost does not contribute to the improvement of the LSSC's comprehensive performance. In the actual time scheduling, LSI may consider using the relationship cost coefficient strategy to improve the comprehensive performance of LSSC, but this improvement is limited.

\subsection{Comparison of the Effects of Different Parameters on LSSC Scheduling Results}

Following the preceding analysis, LSI can use both the relationship cost coefficient and the time delay coefficient to improve the comprehensive performance of LSSC. Indeed, these two strategies are often used by LSIs in actual time scheduling. To compare the effects of these two strategies, we respectively set $C=1.2$ and $R=1.2$ as benchmarks and figure out the proportion of the variations of $Z$ with $R$ and $C$. The results are shown in Table 7 .

Plotting the data in Table 7 into a line chart, we obtain Figure 6. Figure 6 shows the comparison of the performance changes caused by $C$ and $R$. It clearly shows the following.

(1) The slope of the curve of $Z \%$ varied with $R \%$ is bigger than that of $Z \%$ varied with $C \%$. Therefore, compared with the cost augmentation, time delay is better in improving the comprehensive performance of LSSC. 
Table 7: Influence on the comprehensive performance of LSSC caused by time delay and cost increase.

\begin{tabular}{lccc}
\hline$R$ & $Z$ & $R \%$ & $Z \%$ \\
\hline 0.8 & 0.6671 & $-33.33 \%$ & $-2.29 \%$ \\
1 & 0.6802 & $-16.67 \%$ & $-0.37 \%$ \\
$\mathbf{1 . 2}$ & $\mathbf{0 . 6 8 2 7}$ & $\mathbf{0 . 0 0 \%}$ & $\mathbf{0 . 0 0 \%}$ \\
1.4 & 0.6938 & $16.67 \%$ & $1.63 \%$ \\
1.6 & 0.6948 & $33.33 \%$ & $1.77 \%$ \\
1.8 & 0.6972 & $50.00 \%$ & $2.12 \%$ \\
2 & 0.7019 & $66.67 \%$ & $2.81 \%$ \\
\hline$C$ & $Z$ & $C \%$ & $Z \%$ \\
\hline 1.05 & 0.6792 & $-12.50 \%$ & $-0.15 \%$ \\
1.1 & 0.6797 & $-8.33 \%$ & $-0.07 \%$ \\
1.15 & 0.6798 & $-4.17 \%$ & $-0.06 \%$ \\
$\mathbf{1 . 2}$ & $\mathbf{0 . 6 8 0 2}$ & $\mathbf{0 . 0 0} \%$ & $\mathbf{0 . 0 0 \%}$ \\
1.25 & 0.6807 & $4.17 \%$ & $0.07 \%$ \\
1.3 & 0.6809 & $8.33 \%$ & $0.10 \%$ \\
1.35 & 0.6811 & $12.50 \%$ & $0.13 \%$ \\
1.4 & 0.6811 & $16.67 \%$ & $0.13 \%$ \\
1.5 & 0.6811 & $25.00 \%$ & $0.13 \%$ \\
2 & 0.6812 & $66.67 \%$ & $0.15 \%$ \\
\hline
\end{tabular}

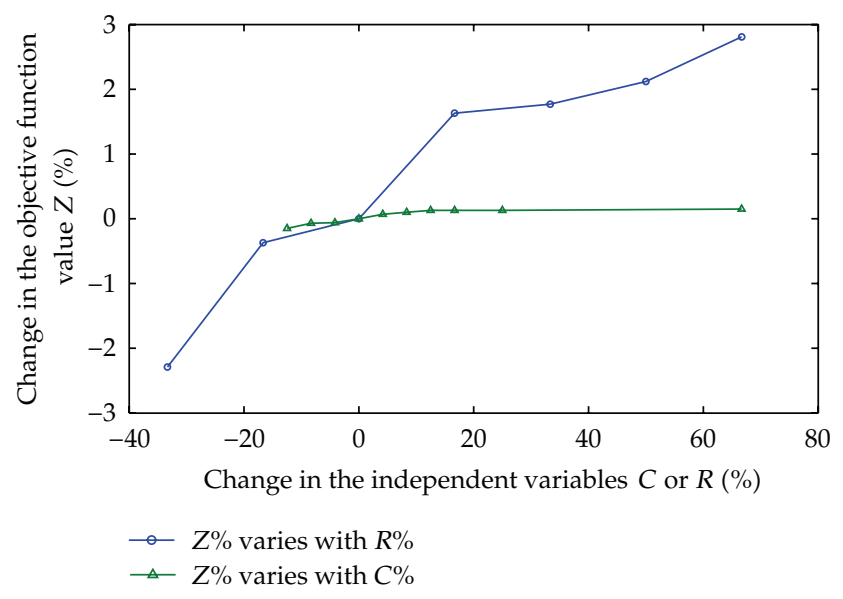

Figure 6: Comparison of the performance changes caused by $C$ and $R$.

(2) In terms of the curve of $Z \%$ varied with $R \%$, when $R \%$ is less than 0 , the slope of the curve is bigger. Thus, if FLSPs operate in the time-compressed state and then the LSI allows some time delay, the improvement in the comprehensive performance of LSSC is much more significant. By contrast, if FLSPs operate in the time delay state, and then the LSI allows more time delay, the improvement in the comprehensive performance of LSSC is not substantial.

\section{Main Conclusions and Management Insights}

Based on the preceding analysis, the following conclusions can be made.

(1) Along with the increase of $R$ (from negative to positive value), the synthesized objective function $Z$ rises gradually, which shows that the overall satisfaction of 
LSSC increases and remains stable upon reaching a certain value with the time delay. Furthermore, time compression decreases the satisfaction level of FLSPs, whereas delaying the completion time increases their satisfaction.

(2) Along with the increase in the LSI's relationship cost coefficient, the comprehensive performance of LSSC increases as well. The effect on improving the comprehensive performance is relatively slow and improvement is limited and remains unchanged upon reaching a certain value.

(3) Both time delay and the LSI's relationship cost can improve the LSSC's comprehensive performance to a certain degree. However, compared with the cost augmentation, time delay is better in improving the comprehensive performance of the LSSC. Based on this result, LSI should address the time scheduling problem reasonably and try to reduce unnecessary time compression requirements to prevent the sharp decline of the LSSC's comprehensive performance. If the comprehensive performance of LSSC has to be improved, LSI should prioritize the use of the time delay strategy.

(4) The results of numerical analysis indicate that the time scheduling model tends to reduce the operation time difference among various FLSPs that are in the same service process and always tries to bring the actual service time close to the expected service time. Thus, it is strongly recommended that the LSI could try its best to choose FLSPs with normal completion times that are close to one another's to minimise the difference in the time of order completion. What the best case scenario is, the normal completion times of those FLSPs are the same as the expected working time. Furthermore, the time scheduling model indicates that with flexible scheduling, we could reach the goals of making the operation time to be compressed or to be delayed in accordance with customer's needs. Time flexibility is a quite important characteristic of flexible supply chain. All the points mentioned previously contribute to make the 3rd-party logistics more reliable to customers, especially the manufacturers. It is helpful to release outsourcing pressure of the manufacturing industry.

(5) Through the model established in this paper, it indicates that it is possible to get an optimized scheduling plan of the FLSPs' operation time if LSI could get the parameters used in the time scheduling model. This model is suitable for the situation which is under the MC background and the CODP has been decided in advance. LSI could use this model to choose a better time delay coefficient and relationship cost coefficient, thus better manage his FLSPs.

\section{Research Limitations and Future Work}

After reviewing the literature on the LSSC scheduling model to minimize the total cost incurred in the LSSC, minimize the difference between the expected and actual times of completing the service order, and maximize the satisfaction of FLSPs, this paper has established a time scheduling model of LSSC that is constrained by the service order time requirement. Numerical analysis has been conducted by using the Matlab 7.0 software. The effects of the relationship cost coefficient and the time delay coefficient on the LSSC's comprehensive performance have been discussed. Finally, the main conclusions and management insights have been presented. 
However, certain limitations remain in this paper. For example, the model solving and analysis are only in accordance with a real numerical example, which cannot represent all actual situations. Besides, we consider the normal service time for a specific order $\left(T_{i j}\right)$ is certain, but in some cases, this parameter is stochastic. In the future, we could continue this research considering the stochastic factors. Furthermore, in this paper, it is assumed that the upstream service capability matches the downstream service capability without considering the unmatched cases. In the future, we can build a time scheduling model in which the capability is matched and the time flexibility is considered. This paper also assumes that mutual trust and collaboration have already been established among the participants in LSSC. However, actual scheduling problems indicate asymmetric information, which should be examined in future research.

\section{Acknowledgments}

This research is supported by the National Natural Science Foundation of China (Grant no. 70902044). The suggestions of the reviewers are also gratefully acknowledged.

\section{References}

[1] C. Chandra and J. Grabis, "Managing logistics for mass customization: the new production frontier," in Proceedings of the 6th Biannual World Automation Congress-Image Processing, Biomedicine, Multimedia, Financial Engineering and Manufacturing-International Forum on Multimedia Image Processing, IFMIP (WAC '04), vol. 18, pp. 335-340, July 2004.

[2] K. L. Choy, C. L. Li, S. C. K. So, H. Lau, S. K. Kwok, and D. W. K. Leung, "Managing uncertainty in logistics service supply chain," International Journal of Risk Assessment and Management, vol. 7, no. 1, pp. 19-22, 2007.

[3] W. H. Liu, X. C. Xu, Z. X. Ren, and Y. Peng, "An emergency order allocation model based on multiprovider in two-echelon logistics service supply chain," Supply Chain Management, vol. 16, no. 6, pp. 390-400, 2011.

[4] C. Y. Lee, S. Piramuthu, and Y. K. Tsai, "Job shop scheduling with a genetic algorithm and machine learning," International Journal of Production Research, vol. 35, no. 4, pp. 1171-1191, 1997.

[5] Y. H. Lee, C. S. Jeong, and C. Moon, "Advanced planning and scheduling with outsourcing in manufacturing supply chain," Computers and Industrial Engineering, vol. 43, no. 1-2, pp. 351-374, 2002.

[6] S. Kreipl and M. Pinedo, "Planning and scheduling in supply chains: an overview of issues in practice," Production and Operations Management, vol. 13, no. 1, pp. 77-92, 2004.

[7] Z.-L. Chen and N. G. Hall, "Supply chain scheduling: conflict and cooperation in assembly systems," Operations Research, vol. 55, no. 6, pp. 1072-1089, 2007.

[8] P. R. Philipoom, "The choice of dispatching rules in a shop using internally set due-dates with quoted leadtime and tardiness costs," International Journal of Production Research, vol. 38, no. 7, pp. 1641-1655, 2000.

[9] N. G. Hall and C. N. Potts, "Supply chain scheduling: batching and delivery," Operations Research, vol. 51, no. 4, pp. 566-584, 2003.

[10] X. G. Xu and X. Y. Li, “Customer order decoupling point selection model in mass customization based on MAS," Journal of Wuhan University of Technology, vol. 28, no. 1, pp. 677-681, 2006.

[11] X. G. Xu, "Position of customer order decoupling point in mass customization," in Proceedings of the 6th International Conference on Machine Learning and Cybernetics (ICMLC '07), pp. 302-307, August 2007.

[12] J. Yao and L. Liu, "Optimization analysis of supply chain scheduling in mass customization," International Journal of Production Economics, vol. 117, no. 1, pp. 197-211, 2009.

[13] J. M. Yao and Y. Pu, "A supply chain scheduling optimization model in mass customization," Systems Engineering, vol. 23, no. 8, pp. 36-41, 2005 (Chinese).

[14] M. Dawande, H. N. Geismar, N. G. Hall, and C. Sriskandarajah, "Supply chain scheduling: distribution systems," Production and Operations Management, vol. 15, no. 2, pp. 243-261, 2006.

[15] N. Mishra, A. K. Choudhary, and M. K. Tiwari, "Modeling the planning and scheduling across the outsourcing supply chain: a chaos-based fast Tabu-SA approach," International Journal of Production Research, vol. 46, no. 13, pp. 3683-3715, 2008. 
[16] J. C. P. Su, Y. L. Chang, M. Ferguson, and J. C. Ho, “The impact of delayed differentiation in make-toorder environments," International Journal of Production Research, vol. 48, no. 19, pp. 5809-5829, 2010.

[17] E. Selvarajah and G. Steiner, "Approximation algorithms for the supplier's supply chain scheduling problem to minimize delivery and inventory holding costs," Operations Research, vol. 57, no. 2, pp. 426-438, 2009.

[18] R. Bhatnagar, P. Mehta, and C. Chong Teo, "Coordination of planning and scheduling decisions in global supply chains with dual supply modes," International Journal of Production Economics, vol. 131, no. 2, pp. 473-482, 2011.

[19] N. Mikati, "Dependence of lead time on batch size studied by a system dynamics model," International Journal of Production Research, vol. 48, no. 18, pp. 5523-5532, 2010.

[20] A. A. Taleizadeh, S. T. A. Niaki, N. Shafii, R. G. Meibodi, and A. Jabbarzadeh, "A particle swarm optimization approach for constraint joint single buyer-single vendor inventory problem with changeable lead time and $(\mathrm{r}, \mathrm{Q})$ policy in supply chain," International Journal of Advanced Manufacturing Technology, vol. 51, no. 9-12, pp. 1209-1223, 2010.

[21] Y. C. Wang, "Evaluating flexibility on order quantity and delivery lead time for a supply chain system," International Journal of Systems Science, vol. 39, no. 12, pp. 1193-1202, 2008.

[22] D. Tang and J. Chen, "Identification of postponement point in service delivery process: A description model," in Proceedings of the 6th International Conference on Service Systems and Service Management (ICSSSM '09), pp. 335-339, June 2009.

[23] C. Jue and T. Daijian, "Modeling the influential factors on determination of CODP in service process," in Proceedings of the International Conference on Management and Service Science (MASS '09), September 2009.

[24] W. H. Liu, J. H. Ji, and L. Zhou, "An order allocation model in two-echelon logistics service supply chain," Journal of Shanghai Jiaotong University, vol. 42, no. 9, pp. 1524-1533, 2008.

[25] B. Manderick, M. K. de Weger, and P. Spiessens, "The Genetic Algorithm and the structure of the fitness landscape," in Proceedings of the 4th International Conference on Genetic Algorithms, pp. 143-150, Morgan Kaufman, San Diego, Calif, USA, 1991. 


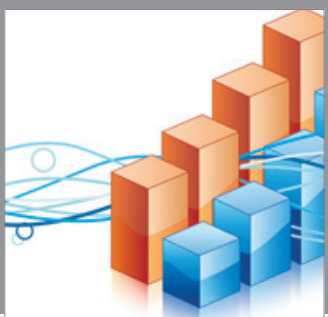

Advances in

Operations Research

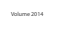

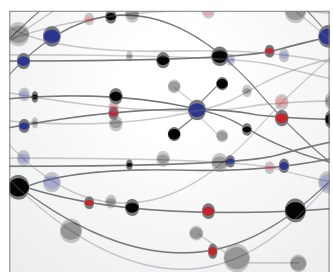

\section{The Scientific} World Journal
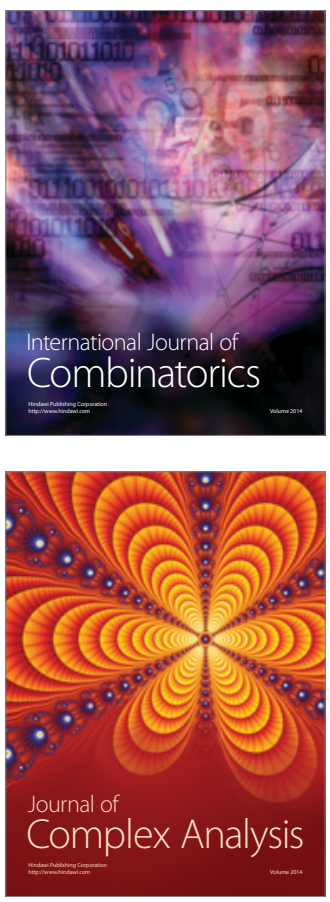

International Journal of

Mathematics and

Mathematical

Sciences
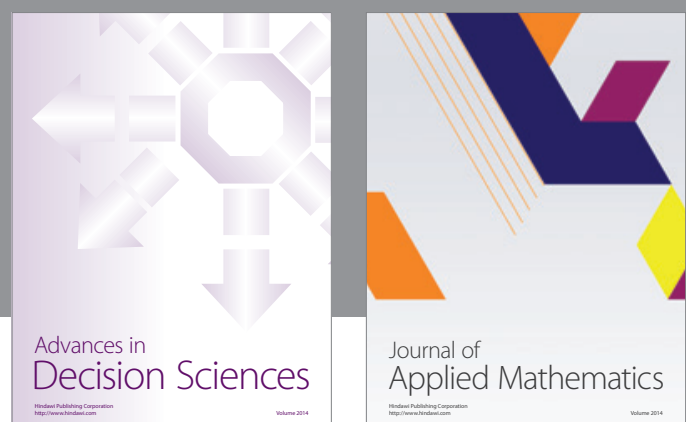

Journal of

Applied Mathematics
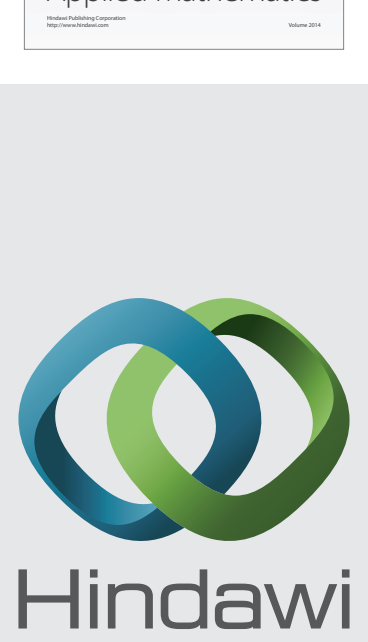

Submit your manuscripts at http://www.hindawi.com
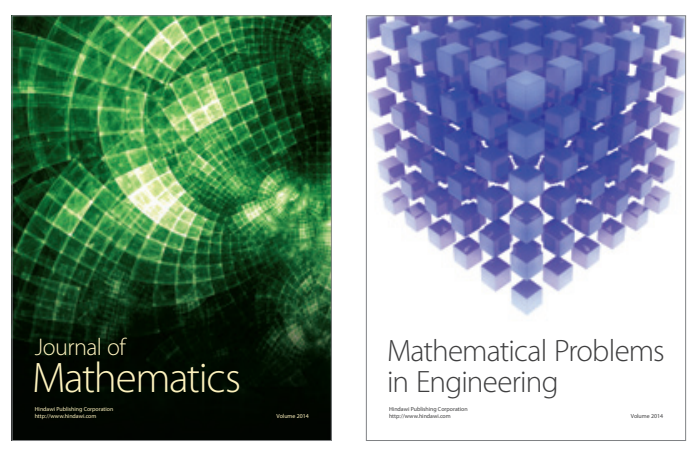

Mathematical Problems in Engineering
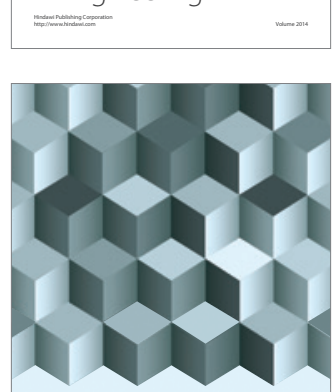

Journal of

Function Spaces
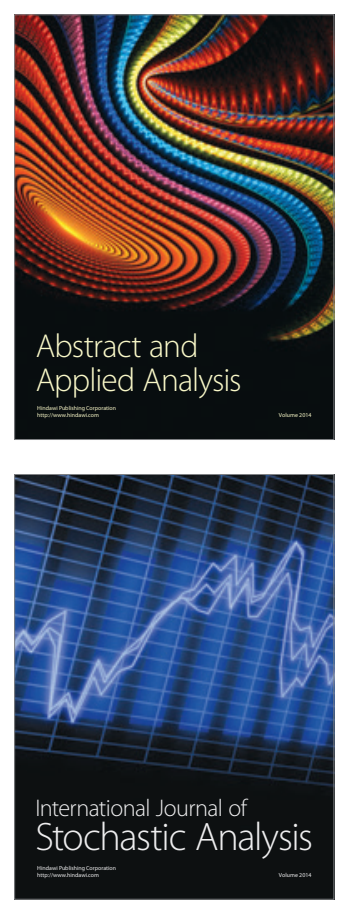

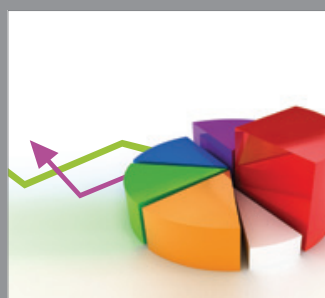

ournal of

Probability and Statistics

Promensencen
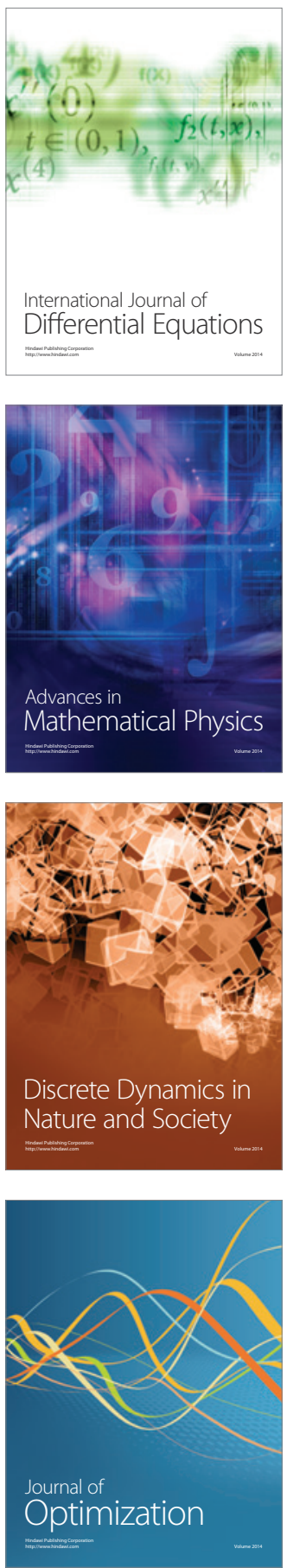